DOI: $10.33947 / 2316-7394-v 8 n 1-3516$

\title{
DESENVOLVIMENTO DE SISTEMA GERENCIADOR DE ESTOQUES FRAGMENTADOS PARA OMNICANALIDADE PARA FIDELIZAÇÃO DO PÚBLICO EM EMPRESAS VAREJISTAS MULTICANAL
}

\section{DEVELOPMENT OF FRAGMENTED INVENTORY MANAGEMENT SYSTEM FOR OMNICHANNEL FOR CLIENTS LOYALTY IN MULTICHANNEL RETAIL COMPANIES}

Tiago Kiritschenko Maffi', Gabriel Santos Nascimento Silva², Erwin Alexander Uhlmann³

\section{RESUMO}

O presente artigo tem a finalidade de demonstrar como é possível construir um sistema capaz de gerenciar estoques em diversos locais, e também auxiliar a tomada de decisão de empresas que atualmente operam sobre o conceito multicanal, fazendo com que atuem na omnicanalidade, integrando seus estoques. A metodologia apresentada no artigo é a utilização de uma loja virtual construída com Wordpress, integrado com um sistema baseado em Codelgniter, utilizando a linguagem PHP e o banco de dados MYSQL, juntamente com a API Distance Matrix da Google. Com base na metodologia do sistema apresentado neste artigo, pontos de venda e centros de distribuições, poderiam ter uma grande influência nos resultados de fidelização de clientes. Reduzindo o tempo de entrega, os estoques estariam melhores distribuídos por oferta e demanda com base em histórico de compras por período. Conclui-se que é uma ferramenta que pode auxiliar as empresas que optarem a utilizá-la para melhorar o relacionamento e assim construir uma melhor fidelidade para com seus clientes.

PALAVRAS-CHAVE: Sistema gerenciador de estoques. Integração de estoques. Sistema omnicanal. Melhoramento de rota. Monitoramento para cadeia puxada.

\begin{abstract}
The purpose of this article is to demonstrate how it is possible to build a system capable of managing inventories in several locations, as well as to help the decision making of companies that currently operate on the multichannel concept, making them act in the omnichannel, integrating their inventories. The methodology presented in the article is the use of a virtual store built with Wordpress, integrated with a system based on Codelgniter, using PHP language and MYSQL database, along with Google's Distance Matrix API. Based on the system methodology presented in this article, points of sale and warehouses could have a great influence on the results of customer loyalty. By reducing delivery time, inventories would be better distributed by supply and demand based on purchase history by a period. It is concluded that it is a tool that can help companies that opt to use it to improve the relationship and thus build a better fidelity to their customers.
\end{abstract}

KEYWORDS: Inventory management system. Inventory integration. Omnichannel system. Route improvement. Monitoring for pulled chain

\footnotetext{
Universidade UNG

Universidade UNG - Docente e Coeditor da Revista Computação Aplicada UNG-Ser
} 


\section{INTRODUÇÃO}

Com clientes cada vez mais conectados, a busca das empresas por melhores serviços e prazos de entrega tem aumentado consideravelmente, envolvendo rápidas variações de demanda, menores e mais frequentes pedidos, elevada competição e reivindicações por melhores níveis de serviço (ROODBERGEN e VIS, 2009).

O Brasil tem avançado nos serviços oferecidos pelas lojas virtuais, porém é necessário muito avanço, para conquistar a confiança e a preferência dos clientes na busca da integração total dos canais. (EBIT, 2016).

O conceito de Omnicanalidade define o futuro do mercado no varejo, tanto nacional quanto internacional, em que os canais de uma marca são integrados para atender o consumidor onde quer que ele esteja. (PIRES, 2013). Com a criação deste novo conceito, nasce também um novo consumidor. Que por sua vez utiliza mais de um canal para realizar suas compras, entre estes canais de compra, a Omnicanalidade faz a integração das lojas virtuais e físicas, fazendo assim com que o cliente possa experimentar todas as formas de interação com a empresa. (PIRES, 2013).

Três em cada quatro consumidores no mundo preferem que os processos de compra, que são desconectados se convertam em uma experiência de Omnicanalidade em um futuro próximo. (SABI$\mathrm{NI}, 2018)$.

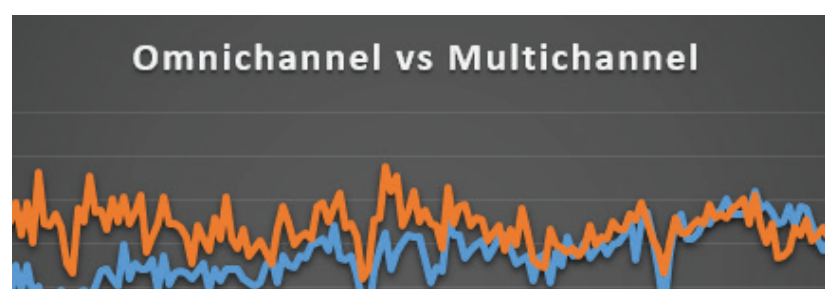

Figura 1: Comparação de termos multichannel e omnichannel. Adaptado de Google Trends, 2018.

Foi realizada uma pesquisa utilizando a ferramenta Google Trends, que mostra de maneira quantitativa, em gráfico de linhas, a quantidade de vezes que um determinado termo foi pesquisado no motor de busca da empresa Google. Para quantificar e embasar a pesquisa, foi-se comparado os termos: $\mathrm{Om}$ nichannel e Multichannel. Logo, pela Figura 1, pode se notar que ao utilizar os filtros: "últimos 5 anos", e local como "Todo o mundo", tem-se o resultado onde as pesquisas estão deixando de ser sobre Multicanalidade e passam a ser sobre Omnicanalidade.

Uma estratégia de Omnicanalidade bem-sucedida, não só garante a sobrevivência do varejista, mas também irá revolucionar a própria indústria de varejo. Os varejistas que conseguem entender que os espaços digitais e físicos em vez de competir entre si, se complementam, terão sucesso. (RIGBY, 2011).

Com um sistema inteligente, as empresas poderiam reduzir o tempo gasto entre a saída do produto da empresa até a chegada à casa do cliente, integrando os pontos de vendas e os centros de distribuições. Imagine que um cliente realiza uma compra em um site, então o pedido dele é integrado ao sistema para que seja feita a retirada do produto dos estoques. Porém, se o sistema entender que perto da residência do cliente existe uma loja, pode fazer com que o pedido do cliente seja retirado desta loja. Fazendo isto, o sistema deve reabastecer a loja da qual foi retirado o pedido.

Esta estratégia poderia contribuir positivamente para a relação entre cliente/empresa e influencia também positivamente no aumento de vendas, já que a entrega seria mais rápida. Neste ponto está a grande diferença, se o produto sair do centro de distribuição até a casa do cliente pode demorar mais tempo.

O objetivo do presente trabalho é demonstrar como é possível construir um software que gerencie estoques fragmentados e também auxilie na tomada de decisão, com isto diminuir o tempo gasto entre a retirada do estoque e a entrega do produto à residência do cliente. Podendo assim, melhorar a experiência que o cliente tem com a empresa, fazendo com que uma oportunidade de fidelização seja construída.

\section{MATERIAIS E MÉTODOS}

O banco de dados pode ser considerado como um armário para arquivamento de dados, porém ele armazena dados do tipo eletrônico. Os registros armazenados em um banco de dados estruturado, são armazenados em tabelas, estas por sua vez são compostas por colunas que são como campos, e assim se formam as linhas de registros. Para ser útil, um usuário desta ferramenta pode solicitar que o sistema faça alterações como incluir, consultar, alterar e remover dados da base (DATE, 2004). A Figura 2 mostra o exemplo de uma pequena tabela, para 
melhor entendimento de como os dados são organizados no banco de dados. A partir destes dados, é possível ter a tomada de decisão, como por exemplo de qual estoque retirar os itens da lista.

\begin{tabular}{c|c|c|c|} 
id_ped & Item_ped & qtd_ped & data_ped \\
\hline 00001 & 12560537 & 1 & $30 / 09 / 2018$ \\
\hline 00002 & 12383520 & 3 & $30 / 09 / 2018$ \\
\hline 00003 & 12384771 & 2 & $30 / 09 / 2018$ \\
\hline 00004 & 12383503 & 5 & $30 / 09 / 2018$ \\
\hline 00005 & 12383430 & 1 & $30 / 09 / 2018$ \\
\hline 00006 & 12387240 & 3 & $30 / 09 / 2018$ \\
\hline 00007 & 12383473 & 4 & $30 / 09 / 2018$ \\
\hline
\end{tabular}

Figura 2: Tabela de pedidos de um e-commerce

Wordpress é definido como um CMS (Content Management System) traduzido para Sistema de Gerenciamento de Conteúdo (RODRIGUES, 2016), suas principais características são: Ambiente administrativo fácil e intuitivo. É utilizado para geração de conteúdo e também para criar uma loja virtual de forma rápida e sem custo.

Para a integração dos pedidos no sistema que irá gerenciar os estoques, utiliza-se o plugin woocommerce, pois tem como função estender as funções do wordpress, para se tornar uma loja virtual e gerar API's que serão enviadas via JSON como pode ser visto na Figura 3.

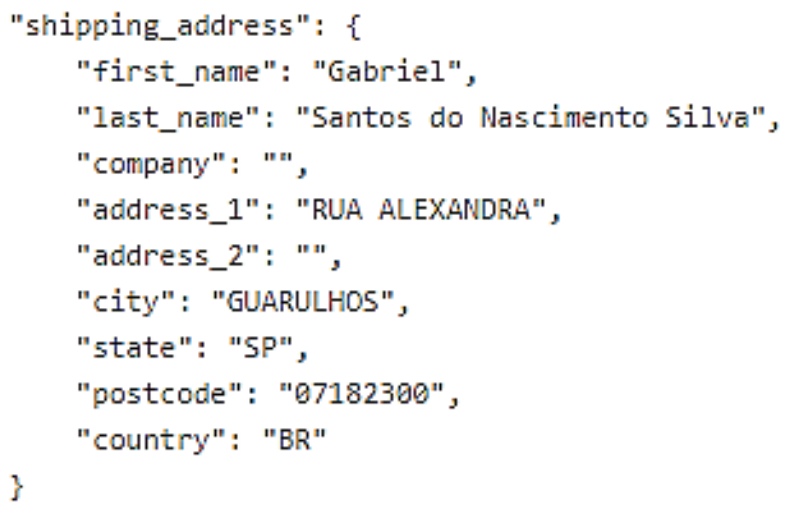

Figura 3: Envio do JSON da ordem do pedido da loja virtual

O Codelgniter é um framework para o desenvolvimento de sistemas web utilizando PHP. É útil para permitir o desenvolvimento de projetos de forma mais rápida e produtiva, quando comparado ao processo de escrever o código manualmente.

Com o Codeigniter podemos facilmente capturar a os dados via JSON, informando a URL da loja virtual e as chaves como mostrado na Figura 4.

\$this->woo_client $=$ new WC_API_Client $($ ' https://inventoryofthings.'Com.'br/ecommerce/', \$this->consumer key, \$this->consumer_secret, \$this->options );

Figura 4: Captura do JSON da ordem do pedido pelo Codeigniter

API Distance Matrix é um serviço de código aberto da empresa Google, que permite acesso via internet. Utilizado em geocodificação, para se obter a distância real entre dois locais, usando como parâmetro a latitude/longitude, nome de rua e CEP. Como exemplo, para o presente projeto, esta API será utilizada no momento da comparação do CEP da casa do cliente com os CEPs das lojas registradas no banco de dados.

Com as informações coletadas sobre o local de entrega do produto, para que seja possível analisar qual o local mais perto e que possuem estoque do item comprado, se faz necessário uma integração com a API utilizando como parâmetro o CEP do cliente e os CEPs das lojas previamente cadastradas na base de dados. A aplicação então vincula no banco de dados o pedido com a loja mais próxima que conseguir do retorno da API. E a partir daí cria uma demanda para esta loja específica retirar o produto do cliente de seu estoque.

O sistema irá analisar os estoques cadastrados procurando algum produto com estoque abaixo do recomendado, caso tenha, é gerado uma ordem de serviço para que o estoque seja reposto. O mesmo também é válido para o cenário onde uma venda é realizada através da loja do e-commerce. O cliente compra o produto no site, e com base na localização do endereço de entrega, o sistema se encarrega de analisar em qual estoque é mais viável retirar o produto. Fazendo isto de uma maneira que agilize a entrega do produto para o cliente. Consequentemente após a ordem de retirada para o cliente ser concluída, o sistema deve criar uma ordem de reposição do produto ao estoque.

A partir do momento que o banco de dados tem o pedido do cliente atualizado e vinculado com a filial mais próxima retornada pela API, o banco de dados utilizará um evento que pode ser programado com base em tempo, ou seja, em uma frequência de minutos executa a ação para criar uma tarefa que será 
atendida pela filial vinculada ao pedido. Este evento levará em consideração o estoque da filial, e também os itens que pertencem ao pedido. Por outro ponto de vista, a loja pode realizar uma consulta na base de dados por meio de uma aplicação do sistema, onde será possível ver os pedidos pendentes de serem retirados do estoque.

\section{RESULTADOS E DISCUSSÃO}

O Omnichannel pode ser definido como a integração de múltiplos pontos de contato com o cliente, com a finalidade de proporcionar uma melhor experiência de compra, unificando as lojas físicas e virtuais (EBIT, 2016). As características da Omnicanalidade são: Constante comunicação entre os canais, política de preços adequada, disponibilidade e detalhes dos produtos. Além de uma comunicação sem quebra entre canais. (CRISTINA, 2015). A estratégia omnicanal pode ser utilizada para realizar uma melhor experiência com o usuário, por meio da personalização da jornada de compra, e assim aumentando o grau de satisfação e fidelização dos clientes (EBIT, 2016). Por exemplo existem diversas empresas que possibilitam que o cliente navegue pelo aplicativo Instagram, veja seus produtos e caso tenha interesse em comprar, é redirecionado à loja virtual onde pode concluir a compra.

O Multichannel ou multicanalidade, pode ser definido como dois ou mais canais de venda, distingue-se do Omnichannel por ter diversos canais, porém sem nenhuma comunicação entre estes (FELIZETTI e GHODOSSI, 2017). Algumas de suas características são: canais independentes, diferentes sensações de compra e um ou mais canais atuam como um concorrente uns dos outros com práticas de preços diferentes. Em muitos casos, os consumidores não são levados a uma experiência integrada, com mensagens consistentes por meio destes canais. É útil para alcançar o público alvo por diversos canais, como por exemplo, a utilização por meio de sites, blogs, aplicativos, mídias sociais e lojas físicas.

A logística é um termo de origem grega logos, definindo a arte do planejamento e estruturação para realização de projetos. Algumas de suas características são: a organização na distribuição dos suprimentos, como armas, comida, transportes, entre outros, para obter uma maior agilidade perante os oponentes (FELIZETTI e GHODOSSI, 2017). A logística é um dos pontos mais importantes quando se trata de operação e planejamento de lojas virtuais, uma vez que pode afetar o resultado financeiro na empresa, além da fidelização e satisfação dos consumidores. (EBIT, 2018). Como exemplo podemos citar a entrega de um produto para o consumidor final, onde passa pelo processo de entrada, separação, movimentação, e retirada do produto.

Um WMS (Warehouse Management System) é um sistema de gerenciamento de estoque utilizado como solução para aumentar a produtividade nos processos, acuracidade e controle de inventário dentro de um armazém (FELIZETTI e GHODOSSI, 2017). Entre as principais características do WMS estão a movimentação de mercadoria, recebimento de produtos, separações expedições, roteirização de picking e cálculo de cartonização de embalagens (ARBACHE, 2011). O WMS pode ser utilizado para a diminuição de custos, e aumento do lucro obtido pela melhoria dos procedimentos internos do armazém (FELIZETTI e GHODOSSI, 2017). Por exemplo, empresas como: Nike, FedEx, Lacoste, DHL e Riachuelo utilizam o WMS Manhattan para processos como recebimento, movimentação, separação e expedição dos produtos nos seus diversos canais.

Com um mundo cada vez mais acelerado é muito importante para uma empresa que invista em formas de acompanhar tal velocidade, inclusive na entrega do produto através de um método que agilize este processo.

Com base nessa metodologia, pontos de venda e centros de distribuições, poderiam ter resultados em fidelização de clientes, reduzindo o tempo de entrega, os estoques estariam melhores distribuídos por oferta e demanda com base em histórico de compras por período.

No pior cenário, o sistema não encontrará uma filial que tenha em estoque o pedido do cliente, fazendo necessário criar uma tarefa para o centro de distribuição fazer a retirada para o cliente.

BOPS (Buy Online Pickup in Store) é um termo de logística que em uma tradução livre, significa compre online retire em loja, muitas vezes resumido como pickup in store. (EBIT, 2018), a característica principal dele é justamente a compra em e-commer$c e$, e a escolha de retirada em lojas. Sua utilidade é trazer facilidade para o cliente que deseja pegar 0 produto na hora e no local onde desejar, por exemplo lojas de e-commerce tem investido muito na integra- 
ção de retirada em loja com omnicanalidade como por exemplo $21 \%$ dos consumidores do Reino Unido usam o modelo de retirada em loja (EBIT, 2018).

Showrooming pode ser definido como o comportamento onde cliente busca o produto na loja física e finaliza a compra pela loja virtual (FAGUNDES, 2018). Sua principal característica é a comodidade em comprar o produto pela internet, é útil para o consumidor quando deseja ver, experimentar e obter mais detalhes com a loja sobre o produto sem ter que comprar na hora, por exemplo ao visualizar o produto por meio da loja física, o consumidor é levado a pesquisar o produto na loja virtual passando assim por uma jornada de compra que começou na loja e terminou pelo e-commerce.

Webrooming é o oposto do Showrooming, o cliente busca o produto na loja virtual, e finaliza a compra na loja física (FAGUNDES, 2018), tem como principal característica uma experiência mais sensorial do produto, é útil para experimentar a mercadoria, escolher o produto pelo preço mais barato e pegar o item diretamente na loja, sem a necessidade de ficar esperando pelo produto ser entregue no local desejado e pagar pelo frete. Por exemplo o cliente pesquisa o produto pelo e-commerce de várias lojas, e vê que na loja perto do trabalho, está o que o consumidor deseja, assim, ao passar pelo trabalho, compra o item, economizando no frete. A Figura 5 informa que $93 \%$ dos consumidores preferem fazer compras online depois de ver o produto em uma loja física, ou seja, tem um comportamento Showrooming, e $82 \%$ têm o comportamento oposto, onde, pesquisam o produto online e compram na loja física, ou seja, tem um comportamento Webrooming.

O Wordpress é uma ferramenta leve, de fácil acesso, porém oferece limitações a empresas que necessitam de um e-commerce mais robusto, ou seja, que tem muita demanda, e necessitam de mão de obra especializada para gerenciar a loja virtual aumentando assim o seu custo (RODRIGUES, 2016). Por isso o Magento é melhor para empresas que necessitam de uma maior estrutura na sua loja virtual.

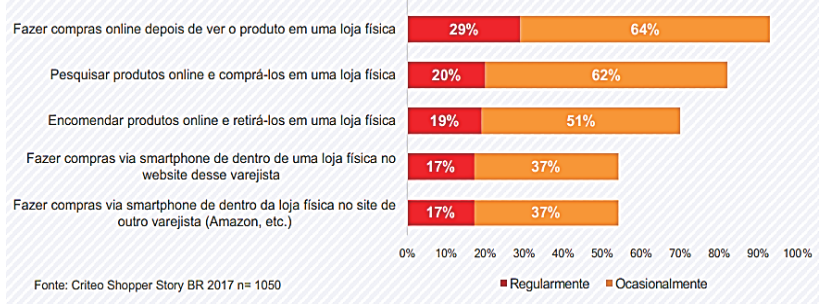

Figura 5: Preferência de compra do consumidor. Fonte: (CRITEO, 2017).

O banco de dados MySQL é um banco de dados relacional, enquanto o Mongo DB não é relacional e sim orientado à documentos, ou seja, o primeiro tem as tabelas relacionadas e cada coluna de suas colunas possuem um tipo, seja referente a armazenagem de texto ou dados numéricos. Já o Mongo DB faz a leitura por meio de arrays que são vetores indexados. (MARQUES et al. 2018). Embora o Mongo DB seja mais rápido nos quesitos de armazenagem e leitura, como mostra Marques, o MySQL conta com programas que permitem utilizá-lo com interface gráfica, fazendo com que a apreensibilidade seja mais fácil, já o MongoDB a utilização é apenas por linha de comando. Sendo assim, este é o motivo da utilização do MySql.

\section{CONCLUSÃO}

Com base no problema de estoques fragmentados, utilizando o método de desenvolvimento de um sistema para gerenciar tais estoques como o apresentado, espera-se que os clientes voltem a comprar na loja através da integração dos canais. Pois com este novo processo, a entrega que a empresa faz do produto ao cliente, pode ser mais rápida.

Sendo assim, o presente projeto tem a finalidade de apresentar uma possível solução que pode auxiliar as empresas a definirem a viabilidade da implantação deste sistema na sua rede empresarial.

Conclui-se que é uma ferramenta que pode auxiliar as empresas que optarem a utilizá-la a melhorar a fidelidade de seus clientes. 


\section{REFERÊNCIAS}

ANGOTTI, Leandro. Omnichannel: uma gestão integrada. 2017, Fundação Getulio Vargas. Disponível em: <http://bibliotecadigital.fgv.br/ojs/index.php/gvexecutivo/article/viewFile/67453/65255> Acesso em: 20 Ago. 2018.

ARBACHE, Fernando Saba et al. Gestão de Logística, distribuição e trade marketing. 4. ed. Rio De Janeiro: FGV, 2011.

CHIANG, LIN e CHEN, et. All. Analysis of Urinary nucle 2011.

CORREIA, J. H.; SOUSA, G. F.; NASCIMENTO, I. Q.; FORMIGA, L. A. C.; NASCIMENTO, R. Q. Otimização e implementação de um sistema de alocação ótima de serviços públicos utilizando a metaheurística Grasp. XLII Simpósio Brasileiro de Pesquisa Operacional, Bento Gonçalves, Brasil, Setembro de 2010. Disponível em: <http://www.din.uem. br/ ademir/sbpo/sbpo2010/pdf/74651.pdf> Acesso em: 13 nov. 2018.

CRISTINA, Sandra. Desafios do omnichannel na aplicação às empresas nacionais. 2015 Faculdade de Engenharia da Universidade do Porto. Disponível em: <https://repositorio-aberto.up.pt/bitstream/10216/80133/2/36379.pdf > Acesso em: 20 ago. 2018.

CRITEO. 2017. The Shopper Story. Disponível em: https://www.criteo.com/br/wp-content/uploads/sites/5/2017/12/TheShopperStory BR.pdf Acesso em: 30 de set. 2018 .

DATE, C. J. Introdução a Sistemas de banco de dados. 8. ed. Editora Campus, 2004. (Capítulos 1 e 2).

E-BIT. 2016. Relatório WebShoppers. São Paulo, ed 33, 2016. Disponível em: http://www.ebit.com.br/ webshoppers?webShopper.periodicityEmail=S\&urID ownloadType=pt BR. Acesso em: 30 set. 2018.
E-BIT, 2018. Relatório WebShoppers. São Paulo, ed 38, 2018. Disponível em: http://www.ebit.com.br/ webshoppers?webShopper.periodicityEmail=S\&urID ownloadType=pt BR. Acesso em: 30 set. 2018.

FAGUNDES, Ligia. As perspectivas do Omnichannel frente à comunicação. 2018 Universidade Metodista de São Paulo. Disponível em: http://tede. metodista.br/jspui/bitstream/tede/1746/2/Ligia\%20 Fagundes.pdf Acesso em: 20 ago. de 2018.

FELIZETTI e GHODOSSI, 2017, Warehouse management system: como solução e fator logístico. Disponível em: https://publicacao.uniasselvi.com.br/ index.php/TI EaD/article/download/1687/801 Acesso em: 30 set. 2018.

FLEURY, Paulo. Supply Chain Management: Conceitos, 1999 Oportunidades e Desafios da Implementação. Disponível em: http://professorricardo.tripod. com/Artigo 15.pdf Acesso em: 30 set. 2018.

MARQUES, Gerson V.; MEDEIROS, Claudio R.; PEREIRA, Jeferson $Q$. Análise comparativa de desempenho de aplicação Java com persistência em Banco de Dados MySQL e MongoDB. 2018. Disponível em: https://periodicos.ufersa.edu.br/index.php/ecop/article/view/7886 Acesso em: 13 nov. 2018.

PIRES, Fábio. 2013. O e-commerce e a era do Omni-Channel. Disponível em: https://www.ecommercebrasil.com.br/artigos/o-e-commerce-e-a-era-do-omni-channel/ Acesso em: 17 ago. 2018.

RIGBY, Darrell. The Future of Shopping. Harvard Business Review, 2011. Disponível em: http://vedpuriswar.org/Readings/The \%20Future $\% 20$ of $\% 20$ Shopping.pdf Acesso em: 18 ago. 2018.

RODRIGUES, Bernardo. 2016. Comércio electrónico e desenvolvimento de websites em WordPress - Relatório de Estágio Disponível em: https:// run.unl.pt/bitstream/10362/19763/1/bernardo\%20rodriques\%20relatorio\%20estagio.pdf/ Acesso em: 13 de nov. 2018 
SORIANO, Felipe; JUNIOR, Alexandre. 2014. Uma análise do sistema de gestão WMS: um estudo multicaso em empresas desenvolvedoras e usuários. Disponível em: https://producaoonline.org.br/rpo/article/view/1582 Acesso em: 30 set. 2018.

SABINI, Walter. 2018. Você está preparado para a omnicanalidade? Disponível em: https://www.ecommercebrasil.com.br/artigos/voce-esta-preparado-para-omnicanalidade/ Acesso em: 17 ago. 2018. 